Association for Information Systems AIS Electronic Library (AISeL)

Wirtschaftsinformatik Proceedings 2005

Wirtschaftsinformatik

February 2005

\title{
Verbreitung, Anwendungsfelder und Wirtschaftlichkeit von XML in Verlagen - Eine empirische Untersuchung
}

\author{
Alexander Benlian \\ Ludwig-Maximilians-Universität München \\ Monica Reitz \\ Ludwig-Maximilians-Universität München \\ Thomas Wilde \\ Ludwig-Maximilians-Universität München \\ Thomas Hess \\ Ludwig-Maximilians-Universität München
}

Follow this and additional works at: http://aisel.aisnet.org/wi2005

\section{Recommended Citation}

Benlian, Alexander; Reitz, Monica; Wilde, Thomas; and Hess, Thomas, "Verbreitung, Anwendungsfelder und Wirtschaftlichkeit von XML in Verlagen - Eine empirische Untersuchung" (2005). Wirtschaftsinformatik Proceedings 2005. 12.

http://aisel.aisnet.org/wi2005/12

This material is brought to you by the Wirtschaftsinformatik at AIS Electronic Library (AISeL). It has been accepted for inclusion in Wirtschaftsinformatik Proceedings 2005 by an authorized administrator of AIS Electronic Library (AISeL). For more information, please contact elibrary@aisnet.org. 
In: Ferstl, Otto K, u.a. (Hg) 2005. Wirtschaftsinformatik 2005: eEconomy, eGovernment, eSociety; 7. Internationale Tagung Wirtschaftsinformatik 2005. Heidelberg: Physica-Verlag

ISBN: 3-7908-1574-8

(C) Physica-Verlag Heidelberg 2005 


\title{
Verbreitung, Anwendungsfelder und Wirtschaftlichkeit von XML in Verlagen - Eine empirische Untersuchung
}

\author{
Alexander Benlian, Monica Reitz, Thomas Wilde, Thomas Hess \\ Ludwig-Maximilians-Universität München
}

\begin{abstract}
Zusammenfassung: Heutzutage sieht sich die Verlagsbranche immer drängender vor die Herausforderung gestellt, das Internet als Distributionskanal einzusetzen, um sich bietende Potenziale aus einer Mehrfachverwertung von Medieninhalten ausschöpfen zu können. Hierfür ist ein effizienter zwischen-wie innerbetrieblicher Inhalteaustausch zur Integration von Print- und Online-Produkten unerlässlich. Die in diesem Zusammenhang immer häufiger diskutierte XML-Technologie scheint für diese Aufgabe sowohl die technischen Voraussetzungen zu erfüllen als auch zu kostenorientierten Vorteilen aufgrund der hohen Automatisierbarkeit gut strukturierbarer Inhalte zu führen. Der vorliegende Beitrag, der sich auf eine empirische Untersuchung im Zeitraum von Februar bis Mai 2004 in Deutschland stützt, versucht zum einen über die tatsächliche Verbreitung, Nutzung und Wirtschaftlichkeit der XML-Technologie in Print-Verlagen Aufschluss zu geben, zum anderen die in der einschlägigen Literatur zu findenden Aussagen zu überprüfen.
\end{abstract}

Schlüsselworte: XML, Print-Verlage, Medien, Diffusion, Wirtschaftlichkeit

\section{Motivation, Zielsetzung und Vorgehensweise}

Durch die rapide Entwicklung digitaler Technologien stehen Medienunternehmen vor der Herausforderung, neue Wege in der Erzeugung, Bündelung und Distribution von Inhalten $\mathrm{zu}$ beschreiten, um der Nachfrage nach integrierten Leistungsbündeln gerecht $\mathrm{zu}$ werden und dadurch Wettbewerbsvorteile zu erzielen [ScHe02, S. 1; Wirt00, S. 238]. Für die nahtlose digitale Integration von Produktions- und Distributionsprozessen sowie der simultanen bzw. zeitversetzten Belegung unterschiedlicher Medienkanäle bietet die eXtensible Markup Language (XML) gerade in Buch- und Zeitschriftenverlagen erhebliche Einsatz- und Kosteneinsparungspotenziale [RaHe01, S. 229]. Im Gegensatz zu Branchen wie z.B. dem (elektronischen) Handel [z.B. Wei+01b] oder Banken [z.B. Wei+03] werden in Print-Verlagen Anwendungsfelder der Markup-Technologie schwerpunktmäßig nicht nur im Datenaustausch zwischen Applikationen oder Unternehmen, sondern ebenso in der Datenspeicherung und -bündelung gesehen [Rawo02, S. 133]. 
Da die Verabschiedung des XML-Standards mittlerweile mehr als sechs Jahre zurückliegt ${ }^{1}$, stellt sich die Frage, inwieweit XML-Technologien die in wissenschaftlichen Publikationen beschriebenen Potenziale bereits erfüllen bzw. sich in der Praxis der Print-Verlage bisher verbreiten konnten. Erste Marktstudien ohne Branchenfokus über die Diffusion von XML-Standards (z.B. ebXML, RosettaNet) prognostizierten eine Marktdurchdringung XML-basierter E-Commerce-Transaktionen von mehr als $40 \%$ bis Ende 2003 [z.B. Mars01]. Aktuellere Studien zum Stand der Diffusion von XML-basierten E-Business-Standards in Industrie und Handel konnten allerdings nur eine langsame Verbreitung insbesondere in solchen Bereichen feststellen, in denen ältere Formate (wie z.B. EDIFACT) die Diffusion von XML nicht behindern [z.B. QuWi03; Wei+01a]. Analoge Ergebnisse großzahlig-empirischer Diffusionsstudien speziell in Print-Verlagen fehlen hingegen bisher gänzlich, obwohl diese interessante Hinweise über den realen technischen sowie ökonomischen Erfolg von XML liefern könnten.

Der vorliegende Beitrag greift diese Forschungslücke auf und prüft die Fragestellung, inwieweit sich XML-Technologien im Print-Bereich bereits etablieren und ob sie die in der Theorie skizzierten technischen und ökonomischen Potenziale bisher erfüllen konnten. Vor diesem Hintergrund werden in einem ersten Schritt die Grundlagen der XML-Technologie sowie ihre Verbreitung, Anwendungsfelder und Wirtschaftlichkeitspotenziale in printorientierten Medienunternehmen vorgestellt. Kapitel 2 des vorliegenden Beitrags versucht somit, die bisher in der Theorie entwickelten Aussagen über den Einsatz von XML in Print-Verlagen verdichtet wiederzugeben. Anschließend erfolgt eine Beschreibung der innerhalb dieser Studie angewandten großzahlig-empirischen Methodik (Kapitel 3), die die eigentlichen Ergebnisse einleitet (Kapitel 4). Der Beitrag endet schließlich mit einer Zusammenfassung der wesentlichsten Erkenntnisse, die anhand der Studie gewonnen werden konnten (Kapitel 5).

\section{Grundlagen zur XML-Technologie und ihrer Nutzung}

\subsection{Die XML-Technologie - ihre Merkmale und Bestandteile}

Bei der eXtensible Markup Language XML handelt es sich um eine textbasierte Auszeichnungssprache zur Beschreibung von Daten bzw. Dokumenten anhand von Strukturinformationen und Metadaten mit dem Ziel, Inhalte zwischen

\footnotetext{
Der XML-Standard 1.0 wurde erstmalig im Februar 1998 vom W3C verabschiedet $[\mathrm{Bra}+00]$.
} 
unterschiedlichen Anwendungen auszutauschen und weiterzuverarbeiten [Wei+98, S. 1; Rawo02, S. 54]. Dabei wird das Grundkonzept verfolgt, Inhalt, Struktur und Layout voneinander zu trennen.

Die technischen Anforderungen, die an den XML-Einsatz bezüglich der Integration bereits existierender Anwendungen und dem zwischenbetrieblichen Inhalteaustausch in Medienunternehmen gestellt werden, wurden von Rawolle in einer früheren Fallstudie [Raw+02, S. 19ff] bereits identifiziert und bewertet. Im Hinblick auf die Systemarchitektur werden der XML-Technologie große Stärken sowohl in der Plattformunabhängigkeit als auch in der Internetfähigkeit und Flexibilität attestiert. Die beim Systembetrieb erforderliche Performanz und Robustheit scheinen hingegen nicht ausreichend gegeben zu sein, im Gegensatz zur zufrieden stellend gewährleisteten Skalierbarkeit an Systemschnittstellen [Raw+02, S. 25ff]. Die meisten der genannten Anforderungen ergeben sich aus den einzelnen Bestandteilen der XML-Technologie, die im Folgenden kurz dargestellt werden.

Ein XML-Dokument besteht aus einem optionalen Prolog, einem oder meist mehreren Elementen, die durch einen selbst definierten Typ gekennzeichnet, evtl. mit Attributen belegt und von jeweils gleich lautenden Start- und Endtags umschlossen sind. ${ }^{2}$ Ein XML-Dokument muss den definierten Regeln einer ihm zugeordneten Document Type Definition (DTD) entsprechen, die die formale Grammatik zur Festlegung der konkret verwendeten Auszeichnungssprache formuliert und somit die Struktur einer bestimmten Dokumentklasse beschreibt [Rawo02, S. 57f; Wei+98, S. 5ff; RoRi01]. Neben der DTD existieren seit Mai 2001 die vom W3C als Recommendation verabschiedeten XML-Schemata, die im Gegensatz zu der strukturorientierten DTD XML-Grammatiken ausdrucksstärker spezifizieren können, was der Implementierung von datenorientierten Anwendungen zugute kommt [Rawo02, S. 61f; Fall01]. Das Layout eines XML-Dokumentes wird dagegen anhand von Stylesheet-Formatvorlagen festgelegt, da durch die selbst definierten Tags kein einheitliches Layout in einer Applikation (z.B. Webbrowser) festgelegt werden kann. Allerdings ist es durch bestimmte Stylesheet-Sprachen (z.B. XSL) möglich, Dokumente in unterschiedlichen Darstellungsformen zu präsentieren [Wei+98, S. 5f]. Zur Speicherung von XML-Dokumenten ist es nahe liegend auf Datenbanktechniken zurückzugreifen, die es ermöglichen, halbstrukturierte Daten auf effiziente Weise speichern, organisieren und abrufen zu können.

\subsection{Die Verbreitung von XML in Print-Verlagen}

Mit dem Auftreten des Internet hat sich für Printunternehmen im Laufe der Zeit die Möglichkeit erschlossen, ihre Angebote auch auf das Online-Medium auszuweiten, das sich als lukrativer Distributionskanal für Medienprodukte behaupten

2 Kommentare oder Verarbeitungsanweisungen sind nicht zwingend, können aber in ein XML-Dokument ebenfalls integriert werden [Rawo02, S. 56]. 
kann. Die XML-Technologie verspricht dabei, aufgrund ihrer zuvor genannten technischen Gegebenheiten den Inhalteaustausch über das Internet zusätzlich zu begünstigen [Bux+01]. Dies mag einer der vielen Gründe sein, warum ein XMLEinsatz besonders im Printmediensektor erörtert wird und an Bekanntheitsgrad zunimmt. Inwieweit sich die Diskussion bereits auf den Technologielebenszyklus und den entsprechenden Adoptionsphasen (Early Adopters, Early Majority, Late Majority und Laggards) [Roge95, S. 257ff; Spe+02, S.64ff] ausgewirkt hat, ist allerdings für den Print-Bereich noch nicht empirisch erforscht worden. Für die Diffusion der XML-Technologie in Verlagen spielen sicherlich die dort möglichen Anwendungsfelder und ökonomischen Potenziale, die in folgenden beiden Abschnitten erläutert werden, eine entscheidende Rolle.

\subsection{Die Anwendungsfelder der XML-Technologie}

Die Anwendungsfelder von XML können generell in die vier Bereiche der Medien-, Funktions-, Zugangs- und Inhalteintegration eingeteilt werden.

Grundsätzlich lässt sich XML als Speicherformat von Inhalten verwenden. Dazu sollten allerdings einige Anforderungen an die jeweiligen semantischen und pragmatischen XML-Strukturinformationen gestellt werden. So ist darauf zu achten, dass Inhalte so anwendungsneutral, aber detailliert wie möglich ausgezeichnet werden. Damit können Inhalte, deren Verwendung noch nicht bestimmt ist, automatisiert weiterverarbeitet werden, was wiederum die strikte Trennung von Inhalt und Layout erfordert [Rawo02, S. 114ff]. XML als Speicherformat stellt laut Rawolle außerdem eine wichtige Voraussetzung für die Medienintegration dar, also die Bündelung verschiedener Medientypen wie Text, Bild, Video und Audio zu einem multimedialen Verbunddokument. Für diese Art von Dokumenten benötigt man einerseits XML-basierte Integrationssprachen, die für die Bündelung und Koordination der einzelnen Inhaltebausteine zuständig sind, sowie Präsentationssprachen, die für eine ansprechende Wiedergabe sorgen [Rawo02, S. 118ff] ${ }^{3}$.

Eine weitere Anwendungsmöglichkeit für XML wird in der Funktionsintegration zur problemlosen Kommunikation zwischen heterogenen Systemen gesehen. Dabei wird meist auf das Remote Procedure Call (RPC)-Prinzip zurückgegriffen, bei dem „entfernte Funktionsaufrufe als Request/Reply-Nachrichten im Sinne des Client/Server-Kommunikationsmodells" [Rawo02, S. 123] abstrahiert werden. Reine XML-Protokolle ${ }^{4}$ kodieren sowohl die Funktionsaufrufe als auch die Antwortnachrichten in wohlgeformte XML-Dokumente, im Gegensatz zu hybriden Protokollen, bei denen nur die Antwort XML-basiert übermittelt wird [Rawo02, S. 123ff].

3 Für die Medienintegration relevante XML-Formate sind z.B. SMIL (Synchronized Multimedia Integration Language) bzw. SVG (Scalable Vector Graphics).

4 Z.B. XML-RPC und SOAP (Simple Object Access Protocol). 
Die Distribution von Inhalten mittels verschiedener Kombinationen von Transportmedium und Endgerät auf der Grundlage von XML-Technologien wird von Rawolle als Zugangsintegration definiert. Häufig ist dabei eine Formattransformation der vorhandenen Inhalte in die jeweilige Präsentationssprache erforderlich, die u.a. mit Hilfe von medienspezifischen Stylesheets wie z.B. XSL erfolgen kann [Rawo02, S. 131f].

Zum Schluss sei noch eine speziell für die Verlagsbranche relevante Funktionalität der XML-Technologie genannt, die als Inhalteintegration bezeichnet und vorwiegend im Bereich der Online Content Syndication bei der zwischenbetrieblichen Integration von Online-Angeboten angesiedelt wird [Andi04]. Bei der Inhalteintegration unterstützt XML die Prozesse zum einen als Datenaustauschformat ${ }^{5}$ für die technische und zum anderen als Auszeichnungsformat von Metadaten ${ }^{6}$ für die semantische Eingliederung der Inhalte in die Zielprodukte [Rawo02, S. 136ff].

\subsection{Wirtschaftlichkeit des XML-Einsatzes in der Verlagsbranche}

Im Folgenden werden die ökonomischen Gesichtspunkte erörtert, die sich vor allem in printorientierten Medienunternehmen mit dem Einsatz von XML ergeben können. In diesem Zusammenhang sollen die betroffenen verlagsspezifischen Kostenarten - mit Schwerpunkt auf dem Leistungserstellungsprozess - näher beleuchtet sowie die in der Literatur durch den Einsatz von XML unterstellten, idealtypischen Veränderungen beschrieben werden. Zur Veranschaulichung werden deshalb im Folgenden sehr gut strukturierbare Inhalte angenommen, die einer Dokumentklasse angehören und deren Layout sich auf alle Zielprodukte automatisiert überführen lässt [Rawo02, S. 165f].

Wie bereits im Abschnitt 2.1 erläutert, sind XML-Dokumente durch DTDs oder XML-Schemata mit Strukturinformationen zu versehen, was für den Leistungserstellungsprozess bedeutet, Strukturdefinitionen dokumentenspezifisch bereit zu stellen und bei Änderungen anzupassen [Rawo02, S. 146]. Da Strukturdefinitionskosten im herkömmlichen Leistungserstellungsprozess kaum vorzufinden sind, lässt sich vermuten, dass die Fixkosten ${ }^{7}$ in diesem Bereich durch die Einführung der XML-Technologie eine Erhöhung erfahren. Variable Strukturdefinitionskosten scheinen aufgrund des mengenunabhängigen Charakters dieser Kostenart eher

5 Hierfür kommen überwiegend das Syndikations-Protokoll RSS (Rich Site Summary) sowie das XML-basierte Kommunikations-Protokoll ICE (Information and Content Exchange) zur Anwendung.

6 Die Auszeichnung von Metadaten erfolgt meist über RDF (Resource Description Framework), dessen Syntax auf XML aufbaut, und Dublin Core.

7 Bei der Betrachtung der variablen und fixen Kosten ist darauf zu achten, dass sie in Abhängigkeit der erfassten und gestalteten Inhaltebündel zu sehen sind. Die Stückkosten beziehen sich auf die Kosten pro Dokument. 
nicht zu existieren. Die anschließende Erfassung der einzelnen Inhalte, ggf. deren Auszeichnung mit semantischen und pragmatischen Elementen und deren Überprüfung sind von fachkundigem Personal durchzuführen. Die daraus entstehenden Erfassungskosten sind demnach zu den personellen Redaktionskosten zu zählen [Rawo02, S. 163; BDZV86, S. 23]. Durch den Einsatz von XML wird erwartet, dass sowohl die variablen als auch die fixen Kosten der Erfassung steigen. Dies hängt mit dem zusätzlichen Aufwand für die Markierung der Inhalte mit XMLbasierten Auszeichnungselementen zusammen, die ein Bearbeiter pro Dokument ausführen muss und bei der späteren Überprüfung ebenfalls zu berücksichtigen hat. Mengenunabhängig gestaltet sich dabei der Mehraufwand für die Erlernung der anzuwendenden DTDs.

Im Leistungserstellungsprozess folgt auf die Erfassung die in der Verlagsbranche immer häufiger praktizierte Mehrfachverwertung von Inhalten [HeSc04], die es notwendig macht, die gespeicherten Inhalte in medienspezifische Präsentationsformate zu konvertieren. Dadurch entstehen sog. Konvertierungskosten [Rawo02, S. 147 und S. 163ff]. Da sich die für die Mehrfachverwertung von Inhalten notwendigen Formattransformationen durch XML automatisieren lassen können, wird angenommen, dass variable Konvertierungskosten sinken, fixe Konvertierungskosten (durch vorherige Programmierung von Transformationssprachen) dagegen steigen. Gelingt es nicht, das Layout automatisiert vollständig zu generieren, so sind weitere manuelle Gestaltungsarbeiten vorzunehmen, bis das Zielprodukt fertig gestellt ist. Hieraus lassen sich Gestaltungskosten ableiten, die den verlagsspezifischen Herstellungskosten zuzuordnen sind [BDZV86, S. 23].

Mit der Einführung der XML-Technologie wird ferner verbunden, dass die vormals überwiegend manuelle Gestaltungsarbeit weitgehend automatisiert werden kann. Dies hätte zur Folge, dass die Gestaltungskosten pro Dokument sinken und mit steigender Anzahl der Zielmedien gegen Null verlaufen. Allerdings müssten mit der Entwicklung der Stylesheets steigende Fixkosten in Kauf genommen werden. In Tabelle 1 sind diese und die vorangehend dargestellten Thesen noch einmal im Überblick dargestellt.

\begin{tabular}{|l|c|c|}
\hline $\begin{array}{l}\text { Verlagsspezifische } \\
\text { Herstellungskosten }\end{array}$ & $\begin{array}{c}\text { Entwicklung der variablen Stück- } \\
\text { kosten }\end{array}$ & Entwicklung der fixen Kosten \\
\hline Erfassungskosten & $\uparrow$ & $\uparrow$ \\
\hline Gestaltungskosten & $\downarrow$ & $\uparrow$ \\
\hline Konvertierungskosten & $\downarrow$ & $\uparrow$ \\
\hline Strukturdefinitionskosten & - & $\uparrow$ \\
\hline
\end{tabular}

Tabelle 1: Erwartete Veränderungen der Kosten beim Übergang vom herkömmlichen zum XML-basierten Vorgehen in Print-Verlagen [Rawo02, S. 166]

Zur graphischen Veranschaulichung werden in den Abbildungen 1 und 2 die aggregierten verlagsspezifischen Herstellungskosten in Abhängigkeit von der An- 
zahl der Dokumente als Kostenkurven dargestellt. Dabei wird die idealtypische Annahme gut strukturierbarer Inhalte dem Gegenpol schlecht strukturierbarer Inhalte gegenübergestellt [RaHe00, S. 30].

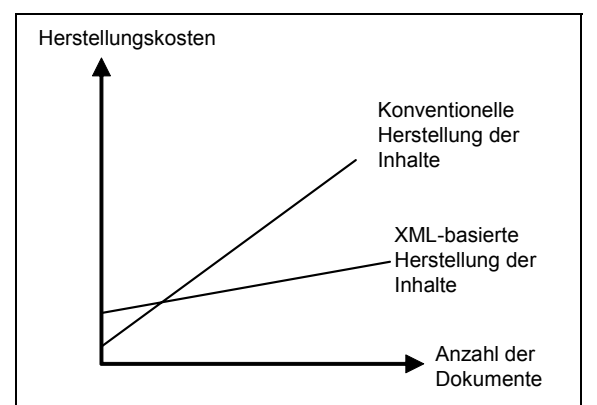

Abbildung 1: Erwartete Kostenstrukturen bei gut strukturierbaren Inhalten

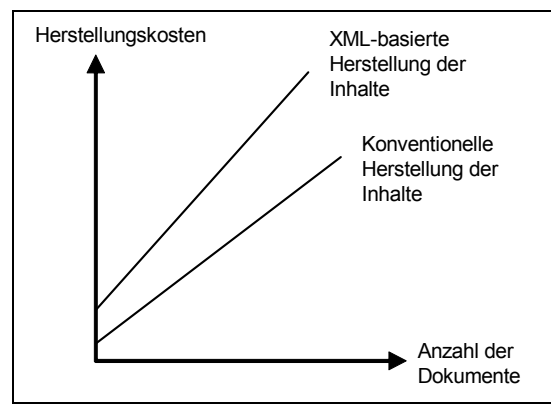

Abbildung 2: Erwartete Kostenstrukturen bei schlecht strukturierbaren Inhalten

Aus Abbildung 1 lässt sich ablesen, dass die Fixkosten beim Übergang zur XMLTechnologie ansteigen, während die variablen Kosten überwiegend sinken ${ }^{8}$, was ab einer bestimmten Auflagenhöhe insgesamt zu einer kostengünstigeren Produktion führt. Dagegen erfahren sowohl die fixen als auch die variablen Kosten bei geringerem Strukturierungsgrad eine Erhöhung, da weder bei der Gestaltung noch bei der Konvertierung die Automatisierbarkeit durch XML vollständig gegeben ist, so dass kostenintensive Nacharbeiten notwendig werden (vgl. Abb. 2). In diesem Fall ist die herkömmliche Herstellungsweise die kostengünstigere.

Für die Wirtschaftlichkeitsbeurteilung des XML-Einsatzes in Verlagen lässt sich insgesamt erwarten, dass sich die variablen Kosten pro Dokument durch strukturierbare Inhalte senken lassen und sich eine steigende Anzahl von Dokumenten positiv auf die Herstellungskostenstruktur auswirkt. Handelt es sich hingegen um Inhalte unstrukturierten Charakters kann angenommen werden, dass Kosten steigernde Faktoren wie die geringere Automatisierbarkeit stärker ins Gewicht fallen, sodass sich die Produktion eher verteuern wird.

\section{Konzeption der Studie}

Das Ziel der durchgeführten empirisch-großzahligen Studie ist die Überprüfung der skizzierten Aussagen über die Verbreitung, typische Anwendungsfelder und Wirtschaftlichkeit der XML-Technologie in Print-Verlagen. Um die in der ein-

8 Es wird angenommen, dass die Kostensenkungen durch die Automatisierung bei der Gestaltung und Konvertierung die Verteuerung des Erfassungsprozesses überkompensieren [Rawo02, S. 168]. 
schlägigen Literatur diesbezüglich aufgestellten Thesen empirisch zu überprüfen, sind die Mitgliedsverlage des Arbeitskreises Elektronisches Publizieren (AKEP) des Börsenvereins des deutschen Buchhandels befragt worden. Da im Rahmen der Erhebung Informationen unterschiedlicher technischer Komplexität und unterschiedlicher Abstraktionsniveaus abgefragt werden sollten, wurde ein mehrstufiges Vorgehen gewählt (vgl. Abb. 3). So konnten in der ersten Phase mit einer relativ großen Stichprobe die Verbreitung von XML untersucht und erste Hinweise auf mögliche Ursachen gesammelt werden. Anhand dieser Daten erfolgte anschließend die Probandenauswahl für die zweite Phase, in der ausschließlich XML-nutzende Verlage bzgl. Anwendungsszenarien und wirtschaftlichen Implikationen befragt wurden. In der dritten Phase, die noch nicht abgeschlossen ist, sollen besonders erfolgreiche XML-Projekte detailliert analysiert werden. Ziel dieser abschließenden Phase ist es, die bisher gewonnenen Erkenntnisse anhand von Fallstudien konkretisieren und Best Practices ableiten zu können.

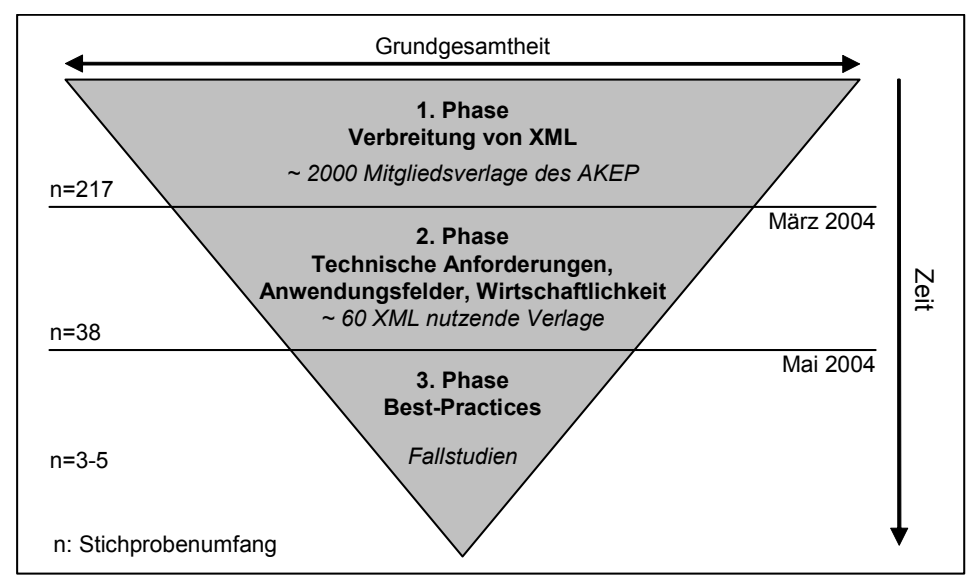

Abbildung 3: Ablauf der Studie

In den ersten beiden Phasen der Untersuchung wurden standardisierte Fragebögen eingesetzt, die vorwiegend Einschätzungen und Erfahrungswerte auf LikertSkalen abfragen. Tiefer gehende Analysen über Prozessstrukturen, Erfolgsfaktoren und Problemfelder eines möglichst idealtypischen XML-Einsatzes erfordern hingegen eher qualitative Methodiken wie etwa Fallstudien, die schließlich in der dritten Phase Anwendung finden sollen.

Die Zusammenstellung und Formulierung des Fragebogens wurden auf Basis der in Abschnitt 2 dokumentierten Literatur und in Zusammenarbeit mit dem AKEP erarbeitet. Der erste Fragebogen hatte zum Ziel, die Stichprobe nach der XML-

9 Der AKEP (siehe http://www.akep.de) versteht sich als eine Informationsplattform, die sich mit den wirtschaftlichen und technischen Anforderungen befasst, die die ITinduzierte Transformation des Verlagswesens mit sich bringt. 
Nutzung sowie weiteren charakteristischen Merkmalen (wie z.B. Verlagsgröße, Medienkanäle sowie Art, Intensität und Dauer von XML-Projekten) zu klassifizieren. In der zweiten Befragung umfasste das Fragebogeninstrument drei Komplexe. Zunächst wurden die Rahmenbedingungen für XML-Projekte (Produktpalette, ITAusstattung) erhoben. Der zweite Fragenkomplex ermittelte Anwendungsszenarien anhand von Zielen und eingesetzten XML-Technologien. Abschließend wurden Implikationen des XML-Einsatzes (Kosten, Prozesse, Integration) sowie eine subjektive Bewertung der Technologie (Zielerreichung, Kosten/Nutzen, Erfolgsfaktoren) abgefragt.

\section{Ergebnisse der ersten Phase}

An der ersten Phase haben, wie in Abbildung 3 beschrieben, 217 von ca. 2.000 Verlagen teilgenommen (Rücklaufquote von ca. 11\%). Nachdem die Stichprobe zunächst insgesamt hinsichtlich ihrer XML-Nutzung untersucht wurde, sollten mit der Aufteilung in die Gruppen der „XML-Nutzer“ (60 Unternehmen) und „NichtXML-Nutzer“ (157 Unternehmen) komparativ-statistische Analysen durchgeführt werden. Zu diesem Zweck konnten die vier Variablen Mitarbeiterzahl (Unternehmensgröße), Anzahl und Art bedienter Medienkanäle sowie die Art der Produktkategorien als differenzierende Merkmale identifiziert werden.

Als erstes Ergebnis der Untersuchung ist festzuhalten, dass heute rund jeder dritte Verlag (ca. 29\%) XML einsetzt. 44\% der Verlage kennen die Technologie, nutzen sie jedoch nicht. Dem verbleibenden Viertel ist XML gänzlich unbekannt (vgl. Abb. 4) ${ }^{10}$.

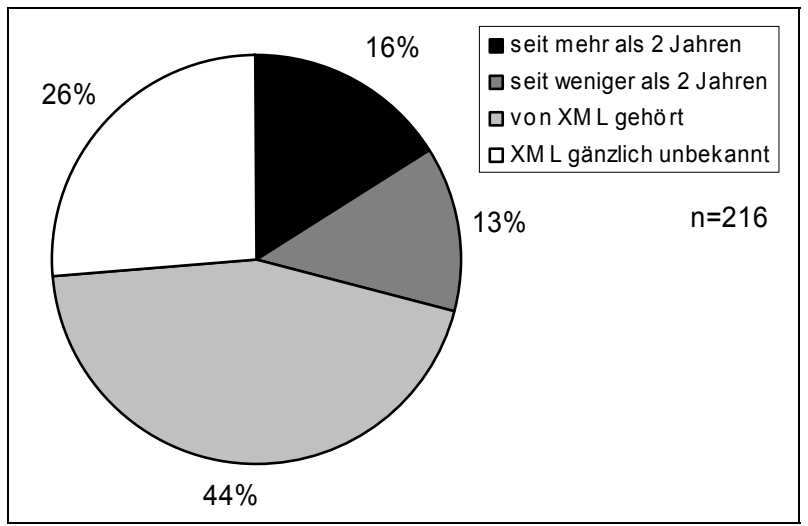

Abbildung 4: Verbreitung und Einsatz von XML

10 Nur 216 gültige Fälle, da 1 missing value 
Der Verbreitungsgrad von XML-Technologien in der Stichprobe lag, nach Angabe der befragten Verlage, vor zwei Jahren bei ca. 16\%, wohingegen dieser heute bereits bei ca. 30\% liegt. Die Verdoppelung der Nutzeranzahl in diesem Zeitraum legt die Vermutung nahe, dass sich der Diffusionsprozess in der Phase der ,early“ oder ,late majority“ befindet. In Anbetracht der Tatsache, dass XML bereits seit 6 Jahren als Standard besteht und als ideale Technologie für den Printsektor angesehen wurde, ist die relativ geringe Verbreitung überraschend.

Zur Charakterisierung der Gruppen der XML-Nutzer und Nicht-XML-Nutzer wurde anschließend die Mitarbeiterzahl herangezogen. Dabei konnte festgestellt werden, dass der überwiegende Teil der großen Verlage ( $>50$ Mitarbeiter) XML anwendet, wohingegen kleinere Verlage die Technologie nur mäßig einsetzen ${ }^{11}$ (vgl. Abb. 5).

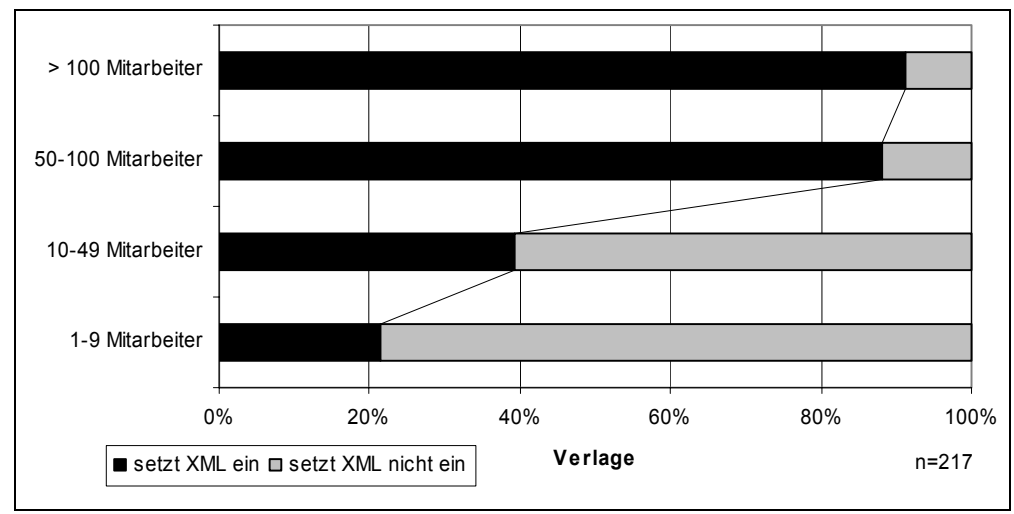

Abbildung 5: XML-Nutzung nach Verlagsgröße

Da die kleinen Verlage aber etwa $75 \%$ der Grundgesamtheit ausmachen ${ }^{12}$, kann das Verhalten der kleinen Verlage als Hauptursache für die insgesamt eher geringe Verbreitung gesehen werden. Als Gründe für den Nicht-Einsatz von XML gaben ca. 38\% der kleineren Verlage fehlendes Know-how und etwa 20\% fehlende Relevanz der Technologie an.

Zur weiteren Unterscheidung der Gruppen soll das Spektrum der bedienten Medienkanäle betrachtet werden (vgl. Abb. 6). Zeitschriften, Loseblattwerke und Kataloge werden etwa in gleichem Umfang von XML-Nutzern wie Nicht-Nutzern verlegt. XML-Nutzer verkaufen Medienprodukte erwartungsgemäß häufiger über „elektronische Medienkanäle“, wohingegen Verlage, die XML nicht einsetzen, eher Buch-Produkte vertreiben.

11 Korrelation zwischen XML-Einsatz (dichotom) und Mitarbeiterzahl: $\mathrm{r} \approx 0,56$, zweiseitig signifikant auf $1 \%$-Niveau

12 Dies ist sowohl in dieser Stichprobe als auch in früheren Erhebungen des AKEP der Fall. 


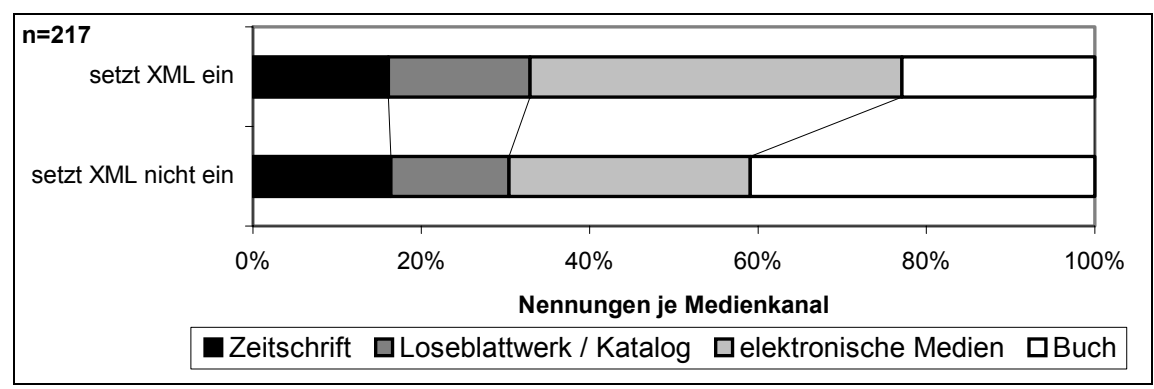

Abbildung 6: Zusammensetzung der Gruppen nach Medienkanälen

Demnach ist die Aktivität im Bereich „elektronische Medien“ bzgl. des durchgeführten Gruppenvergleichs ein relevantes Unterscheidungsmerkmal. Ein Grund hierfür könnte sein, dass XML das volle Potenzial erst bei digitalen Endprodukten entfaltet, da in diesem Bereich ein extrem hoher Automatisierungsgrad erreicht werden kann. Ein anschauliches Beispiel hierfür ist die Generierung von mehreren medienspezifischen digitalen Online-Formaten (z.B. HTML, WML) aus einem medienneutralen Zwischenprodukt unter Verwendung von XSL bzw. XSLT.

Im Rahmen der Verbreitungsbetrachtung würde sich die Verlegertätigkeit im Bereich elektronische Medien damit positiv auf das Adoptionsverhalten auswirken. Diese Annahme wird durch die beobachtete Korrelation zwischen „XML-Einsatz“ (dichotom) und „Einsatz elektronischer Medien“ (dichotom) von 0,414 gestützt. ${ }^{13}$

Bricht man diese Betrachtung auf die Ebene der verlegten Produkte herunter, lässt sich die in Abbildung 7 präsentierte Rangliste von Produktkategorien aufstellen, die den Anteil der XML-nutzenden Verlage je Produktkategorie widerspiegelt.

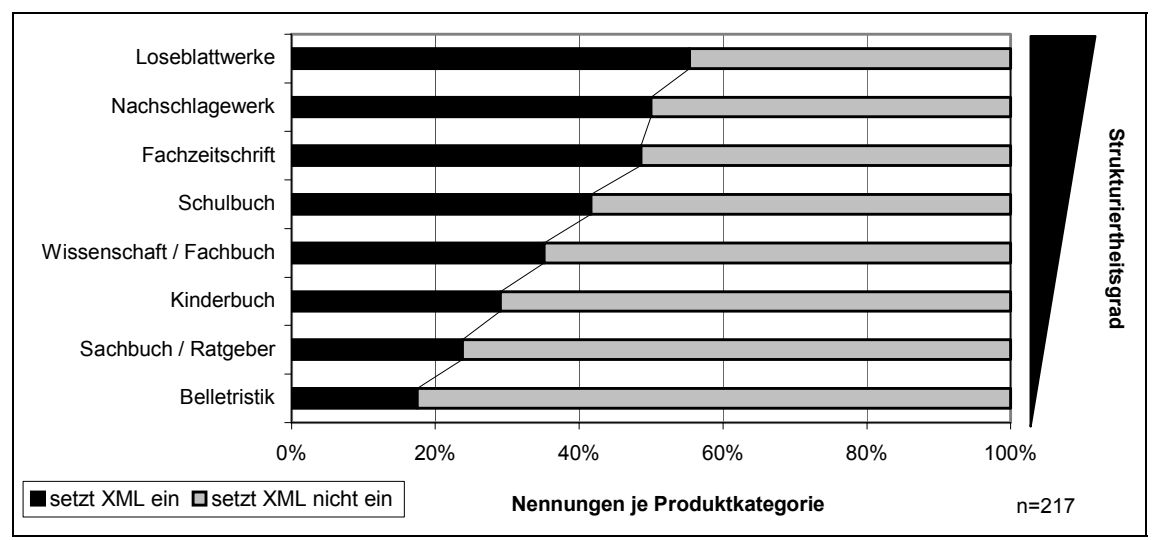

Abbildung 7: XML-Einsatz in den einzelnen Produktkategorien

13 Zweiseitig signifikant auf 1\%-Niveau 
Schätzt man je Produktkategorie einen „durchschnittlichen Strukturiertheitsgrad“ der Inhalte, fällt auf, dass der Anteil der XML-Nutzer bei eher strukturierten Produkten tendenziell höher ist als bei unstrukturierten Produkten. Dies stützt die in der Literatur vertretenen Thesen und spricht dafür, die „Strukturiertheit der Produkte" als weiteren Einflussfaktor auf das Adoptionsverhalten anzusehen. Da in der ersten Phase hierzu keine weiteren Daten erhoben wurden, wird dieser Sachverhalt in der zweiten Phase näher untersucht (siehe 5.3).

Ein weiterer Faktor mit signifikantem ${ }^{14}$ Differenzierungspotenzial ist die Anzahl der bedienten Medienkanäle eines Verlags (vgl. Abb. 8).

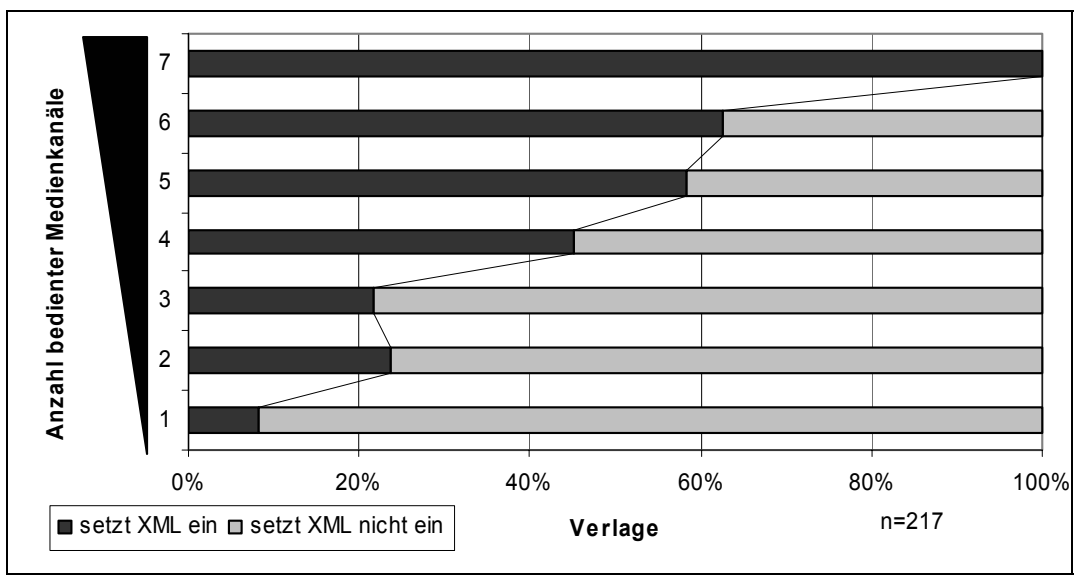

Abbildung 8: XML-Einsatz nach Anzahl der bedienten Medienkanäle

Auch hier kann angenommen werden, dass sich der Einsatz der XML-Technologie effizienter und effektiver gestaltet, wenn mehrere Medienkanäle bedient, d.h. mögliche Potenziale durch eine Mehrfachverwertung von Medieninhalten (z.B. Cross-Media-Publishing) ausgeschöpft werden. Mit einer Korrelation ${ }^{15}$ von 0,473 zwischen „XML-Einsatz“ und „Anzahl bedienter Medienkanäle“ ist dieser Faktor als weitere Einflussgröße auf das Adoptionsverhalten und damit die Diffusion von XML aufzuführen.

14 Mittelwertvergleich, Signifikanzniveau: 1\%, Korrelation zwischen XML-Einsatz (dichotom) und Anzahl der Medienkanäle: $r \approx 0,45$

15 Zweiseitig signifikant auf 1\%-Signifikanzniveau (approximativer Gauß-Test). 


\section{Ergebnisse der zweiten Phase}

Wie bereits in Abschnitt 3 skizziert, wurde in Phase zwei nach technischen Anforderungen, Anwendungsfeldern und Wirtschaftlichkeit der XML-Technologie gefragt. An der zweiten Phase haben 38 der 60 XML-nutzenden Verlage aus der ersten Phase teilgenommen (Rücklaufquote von ca. 63\%). Aufgrund des eher kleinen Stichprobenumfangs wurden zur Beschreibung der Ergebnisse der Phase 2 rein deskriptive Auswertungen durchgeführt, deren Aussagen dennoch einen gewissen Tendenzcharakter aufweisen.

\subsection{Ergebnisse zu technischen Anforderungen und Erfolgsfaktoren von XML}

Das Zufriedenheitsprofil bezüglich der in 2.1 genannten technischen Anforderungen (Plattformunabhängigkeit, Robustheit, Performanz, Skalierbarkeit und Flexibilität) stellt sich in Abbildung 9 dar. Generell ist festzuhalten, dass XML die gestellten Anforderungen tendenziell erfüllt. Mit einem Modalwert von „neutral“ schneiden Skalierbarkeit und Performanz am schlechtesten, die Plattformunabhängigkeit mit ca. 32\% zufriedener und über $47 \%$ vollkommen zufriedener Anwender am besten ab, was mit den bisherigen Annahmen (vgl. 2.1) überwiegend übereinstimmt.

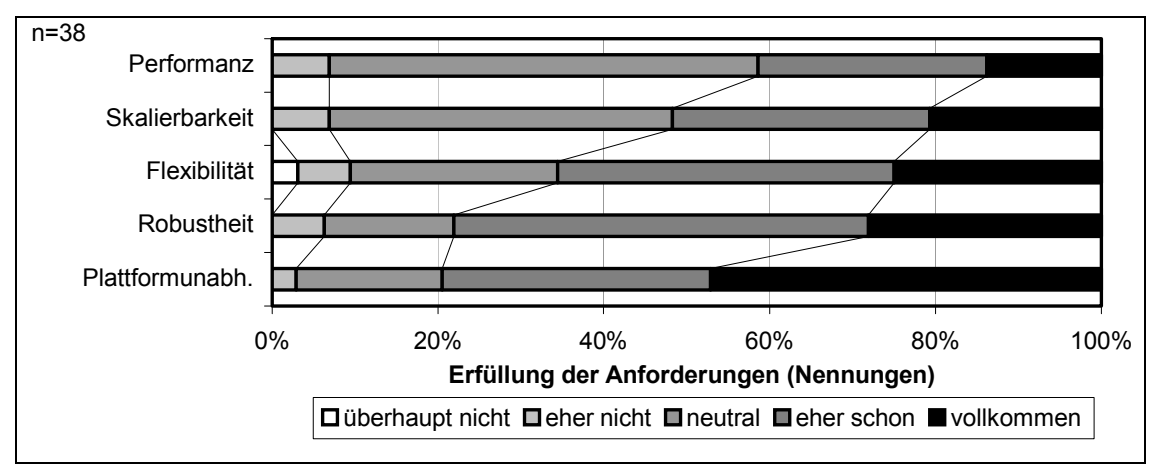

Abbildung 9: Erfüllungsgrad der technischen Anforderungen durch XML

Standardisiertes Format, strukturierte Daten, statisches Layout und automatisierbare Aufgabe wurden in Kapitel 2 als Erfolgsfaktoren für die XML-Nutzung in Verlagen dargestellt. Die Ergebnisse unserer Analysen bestätigen die in der Literatur zu findenden Aussagen. Als eher bzw. vollkommen kritisch für den Erfolg einer XML-Lösung sehen ca. $94 \%{ }^{16}$ der Teilnehmer die Strukturiertheit der Daten

16 Hierbei wurden die Prozent-Werte der Kategorien „eher schon“ und „vollkommen“ addiert. 
an, welche sich damit stark von den anderen Faktoren abhebt. An zweiter Stelle wird die Automatisierbarkeit der Aufgabe mit ca. 76\% als eher bzw. vollkommen erfolgskritisch eingestuft, gefolgt vom standardisierten Format (ca. 69\%) und dem statischen Layout mit etwa 66\%. In Abbildung 10 sind die Ergebnisse im Überblick dargestellt.

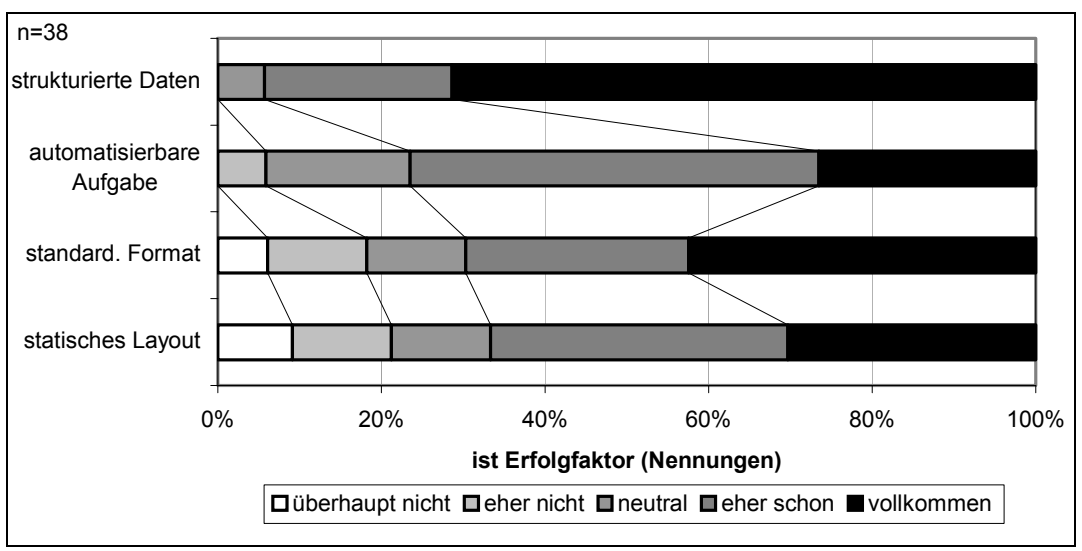

Abbildung 10: Erfolgsfaktoren der XML-Technologie

\subsection{Ergebnisse zu den Anwendungsfeldern von XML}

Die Teilnehmer wurden auch danach befragt, welche der in Kapitel 2.1 beschriebenen Standardbestandteile von XML in der Verlagsbranche tatsächlich Anwendung finden. Erwartungsgemäß wurden DTDs (ca. 87\%) und Stylesheets (ca. $65 \%$ ) als die meist genutzten Technologien genannt, wohingegen Schemata und XML-Datenbanken jeweils zu 42\% und 47\% genutzt werden (vgl. Abb. 11).

Anhand XML-basierter Technologien lassen sich für Print-Verlage verschiedene Funktionalitäten bzw. Anwendungsmöglichkeiten realisieren (vgl. 2.1 und 2.3). Hierbei ist von Interesse, für welche Tätigkeiten XML vornehmlich eingesetzt wird. Während die Formatintegration bzw. Zugangsintegration ${ }^{17}$ mit ca. $81 \%$ am häufigsten genannt wurde, schneidet die Möglichkeit der Funktionsintegration mit ca. 44\% am schlechtesten ab (vgl. Abb. 12). Die Verwendung von XML zur medienneutralen Datenhaltung, die sich aus der in 2.3 erwähnten Speicherfunktionalität sowie der Medienintegration ergibt, kommt ebenso wie der XML-basierte Datenaustausch (als Bestandteil der Inhalteintegration) bei 63\% der Verlagen zum Einsatz. Die für besagte Inhalteintegration ferner notwendige Auszeichnung von Metadaten (vgl. 2.3) wird dagegen nur von jedem zweiten Verlag angewandt.

17 Die in 2.3 beschriebene Zugangsintegration wurde bei dieser Erhebung zum besseren Verständnis als Formatintegration bezeichnet. 


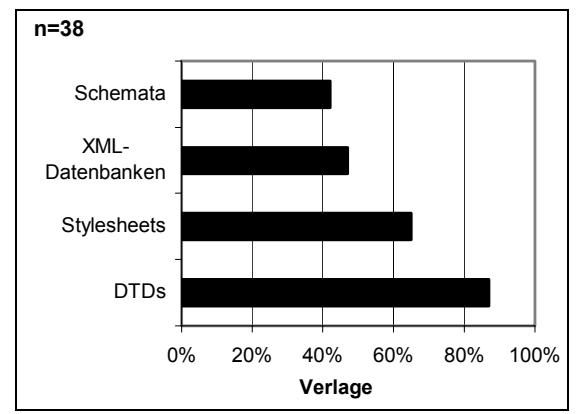

Abbildung 11: Nutzung bestimmter Technologiebestandteile von XML

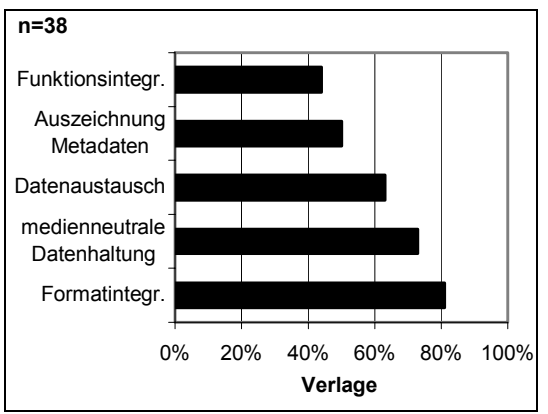

Abbildung 12: Genutzte Funktionalitäten von XML

Wie in 2.3 festgehalten, wird in der Literatur die Inhalteintegration schwerpunktmäßig in Verbindung mit Content Syndication gesehen, was in unserer Analyse tendenziell bestätigt werden konnte (vgl. Abb. 13). Einerseits werden bei hohem Einsatz von XML als Datenaustauschformat in allen beobachteten Fällen Inhalte mehrfach verwertet, andererseits sind bei abnehmender Nutzungsintensität tendenziell auch geringere Aktivitäten bezüglich der Mehrfachverwertung zu verzeichnen. ${ }^{18}$ Dagegen lässt sich in Abbildung 14 allenfalls eine minimale Abhängigkeit zwischen XML als Auszeichnungsformat von Metadaten und der Mehrfachverwertung von Inhalten erkennen.

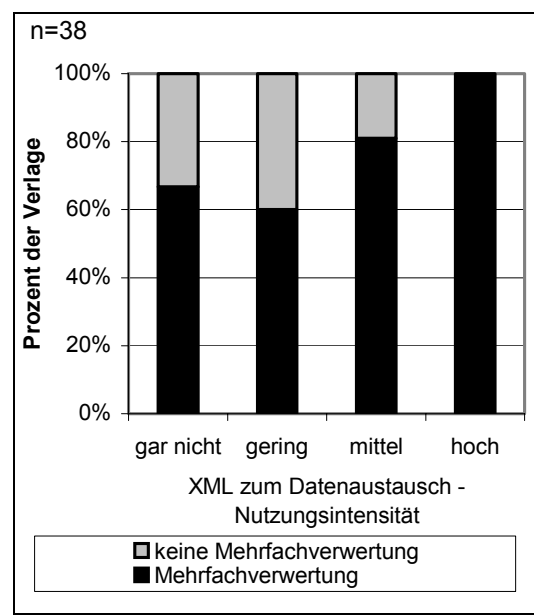

Abbildung 13: XML als Datenaustauschformat $\Leftrightarrow$ Mehrfachverwertung

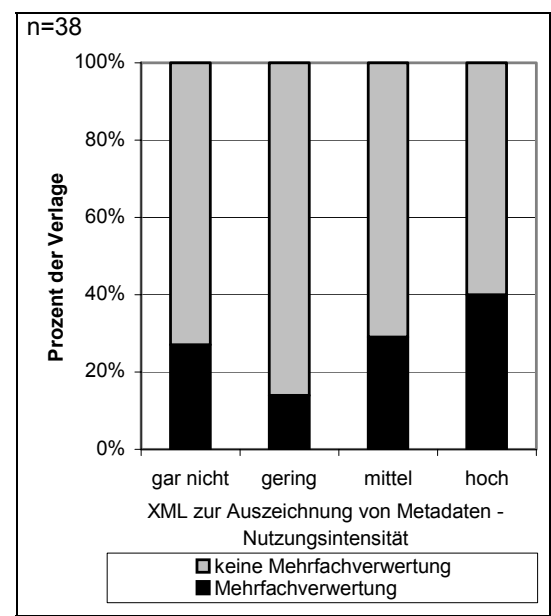

Abbildung 14: XML als Auszeichnungsformat $\Leftrightarrow$ Mehrfachverwertung

18 Die Verzerrung bei ,gar nicht“ ist vermutlich auf die geringe Gesamtzahl derjenigen Verlage zurückzuführen, die XML nicht zum Datenaustausch verwenden. 
Der Zusammenhang zwischen der Zunahme der (Online) Content Syndication bzw. der Mehrfachverwertung von Inhalten im Allgemeinen und dem Einsatz der XML-Technologie lässt darauf schließen, dass XML tatsächlich die Distribution von Medienprodukten unterstützt und mit dem Medium Internet für Print-Verlage neue Geschäftsmodelle generiert.

\subsection{Ergebnisse zur Wirtschaftlichkeit von XML}

Eine allgemeine Kosten-Nutzen-Einschätzung ergab, dass 57\% der in Phase 2 befragten Verlage den Nutzen durch die Einführung der XML-Technologie höher einstufen als die dadurch entstandenen Kostensteigerungen, während insgesamt nur 2 der 37 Verlage $^{19}$ angaben, der Nutzen kompensiere nicht die aufgetretene Verteuerung. Ein relativ hoher Anteil (38\%) erklärt, sich noch unsicher in Bezug auf die Wirtschaftlichkeit von XML zu sein, was unter anderem von der meist kurzen Anwendungsdauer der Technologie im Unternehmen herrühren mag (vgl. Abb. 15).

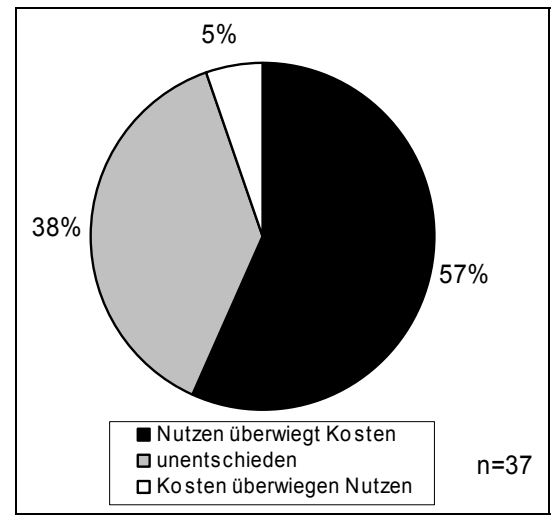

Abbildung 15: Allgemeine Kosten-Nutzen-Einschätzung zum XML-Einsatz

Neben den in Kapitel 2.4 vorgestellten verlagsspezifischen Kostenarten wurden bei der Erhebung auch klassische Kostenarten berücksichtigt, um einen umfassenden Einblick in XML-induzierte Kostenänderungen zu erlangen. Wie Abbildung 16 aufzeigt, weisen abgesehen von den Konvertierungskosten sämtliche anderen Kostenarten teilweise sogar erhebliche Steigerungen auf, wobei die Erfassungsund die Strukturdefinitionskosten die Spitze bilden. Da es sich um Verlage mit meist heterogenem Produktportfolio handelt, also strukturierbare wie unstrukturierbare Inhalte zu bearbeiten sind, ist die starke Senkung der Konvertierungskosten als auch die geringe Verteuerung der Gestaltungsarbeit ein erstaunliches Er-

19 Nur 37 gültige Fälle, da 1 missing value 
gebnis. Dies mag ein Indiz dafür sein, dass der Automatisierungsgrad als kostenkritischer Faktor (vgl. 2.4) bei der Konvertierung und Gestaltung durch den Einsatz der XML-Technologie dennoch weitestgehend gewährleistet zu sein scheint.

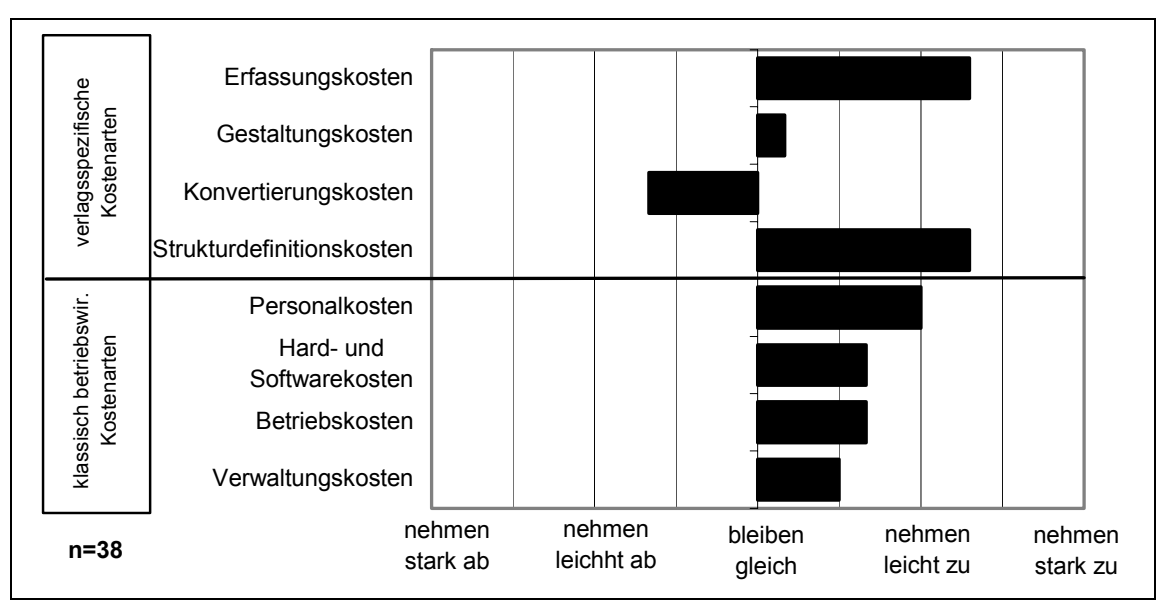

Abbildung 16: Veränderungen in der Kostenstruktur durch XML

Bringt man die oben genannten verlagsspezifischen Kostenarten mit dem Strukturierungsgrad der verwendeten Medieninhalte in Zusammenhang (vgl. Abb. 17), lässt sich - bis auf die Gestaltungskosten - tendenziell eine negative Korrelation feststellen: Je strukturierter die Inhalte sind, desto geringer sind die verlagsspezifischen Herstellungskosten. Dies bestätigt nochmals die Kosten beeinflussende Wirkung des Strukturierungsgrades von Medieninhalten.

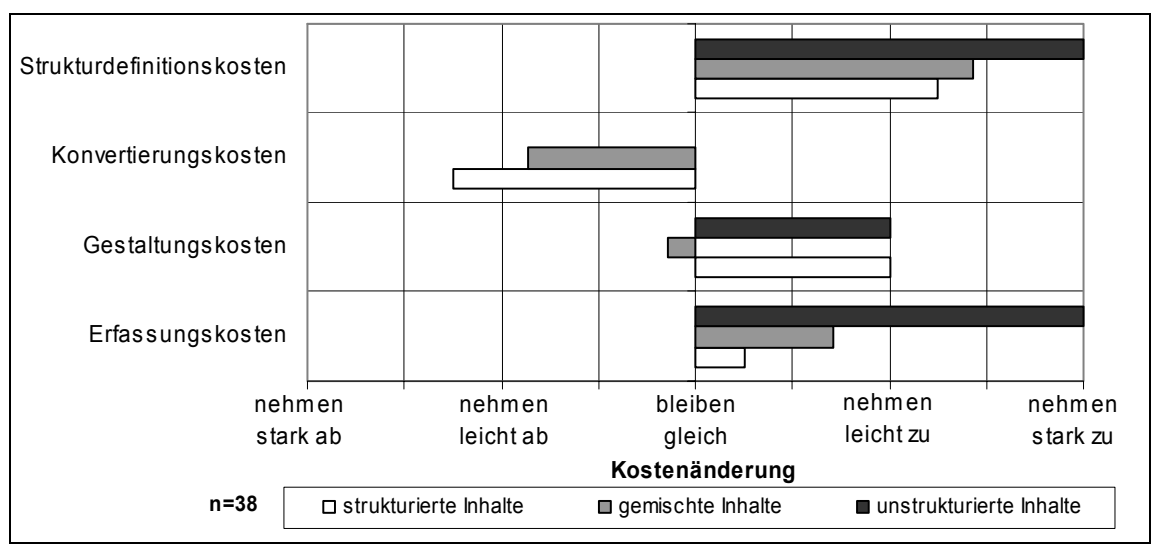

Abbildung 17: Kostenänderungen bei unterschiedlichem Strukturierungsgrad der Inhalte

Da sich die befragten Verlage letztlich weitgehend positiv über die Wirtschaftlichkeit des XML-Einsatzes geäußert haben (vgl. Abb. 15), kann vermutet werden, 
dass sich zum einen (variable) Konvertierungskostendegressionen, zum anderen Umsatzsteigerungspotenziale stark positiv auswirken. Ein Indiz für die Senkung variabler Konvertierungskosten könnte sein, dass $71 \%$ der in der zweiten Runde befragten XML-nutzenden Verlage mehr als 50 Mitarbeiter beschäftigen und somit zu Medienunternehmen zu rechnen sind, die hohe Auflagenzahlen produzieren. Als Indikatoren der Erlössteigerung sehen ca. 58\% der befragten Verlage im XML-Einsatz die Möglichkeit, neue Geschäftsfelder erschließen zu können, ca. 69\% sogar eine Flexibilisierung in der Produktentwicklung.

Auf Basis dieser Ergebnisse kann resümierend festgehalten werden, dass eine bloße unternehmensinterne Kostenperspektive auf den Einsatz von XML zu kurz greift. Weitergehende Untersuchungen, die neben der Kosten- explizit eine Marktperspektive integrieren, können hierbei tiefer gehende Einsichten in die Wirtschaftlichkeit eines XML-Einsatzes liefern.

\section{Fazit}

Mit dieser Studie konnte ein erster empirischer Einblick in die Potenziale und Risiken des Einsatzes der XML-Technologie in printorientierten Medienunternehmen gewonnen werden.

Als Ergebnis zur Diffusionsbetrachtung ist festzuhalten, dass jeder dritte Verlag XML bereits einsetzt und die Technologie weiteren $45 \%$ immerhin bekannt ist. Aufgrund der Verdoppelung der Zahl der XML-Nutzer in den letzten zwei Jahren und dem nunmehr 6-jährigen Bestehen von XML als W3C-Standard ist davon auszugehen, dass sich die Diffusion der Technologie bereits in einem fortgeschrittenen Stadium befindet. Ein positiver Einfluss von Mitarbeiterzahl, Strukturierungsgrad der Produkte und Anzahl der bedienten Medienkanäle auf die XMLNutzung in Print-Verlagen konnte nachgewiesen werden. Ebenso konnte gezeigt werden, dass XML-Nutzer eher elektronische Medien einsetzen als Nicht-XMLNutzer.

Im Weiteren wurden technische Anforderungen an die XML-Technologie untersucht. Die Ergebnisse stimmen größtenteils mit den in einer früheren Fallstudie beobachteten Sachverhalten bzgl. Plattformunabhänigkeit, Robustheit, Performanz, Skalierbarkeit und Flexibilität überein. Insbesondere die Plattformunabhängigkeit wird allgemein als essentielles Merkmal eingestuft. Als Erfolgsfaktor für XML-Projekte konnte hauptsächlich der Strukturierungsgrad der Daten, aber auch die Automatisierbarkeit der Aufgabe identifiziert werden.

Darüber hinaus konnte der vermutete positive Zusammenhang zwischen der Mehrfachverwertung von Inhalten und dem Einsatz von XML zum Datenaustausch und zur Auszeichnung von Metadaten tendenziell bestätigt werden. Durch die fortschreitende Syndizierung von Inhalten - auch auf unternehmensexterner 
Ebene - scheint es mit Hilfe der XML-Technologie daher auch denkbar, dass sich in Zukunft Effizienzsteigerungen zwischen den Wertschöpfungsketten printorientierter Medienunternehmen generieren lassen und sich in der Folge sogar strategische Verlagsnetzwerke herausbilden.

Insgesamt gaben 57\% der befragten Verlage an, dass der durch den Einsatz der XML-Technologie gestiftete Nutzen die Kosten überwiegt. Bei genauerer Betrachtung zeigt sich, dass die Konvertierungskosten durch den Einsatz von XML zurückgehen, wohingegen die Erfassungs- und Strukturdefinitionskosten in Übereinstimmung mit den Aussagen in der Literatur zunehmen.

Abschließend ist zu sagen, dass sich die erhofften Vorteile aus dem Einsatz der XML-Technologie für die Verlagsbranche - zumindest für die befragten Unternehmen - bereits abzuzeichnen beginnen. Es ist allerdings noch abzuwarten, wie sich die XML-Technologie angesichts fortschreitender Standardisierungsbemühungen und der Integration in Content Management Systeme künftig verbreiten wird.

\section{Literatur}

[Andi04] Anding, M.: Online Content Syndication - Theoretische Fundierung und praktische Ausgestaltung eines Geschäftsmodells der Medienindustrie. Gabler, Wiesbaden, 2004.

[BDZV86] BDZV - Bundesverband Deutscher Zeitungsverleger e.V. (Hrsg.): Kostenrechnung für Zeitungsverlage. Bonn, 1986.

[Bra+00] Bray, T.; Paoli, J.; Sperberg-McQuenn, C. M.; Maler, E.: Extensible Markup Language (XML) 1.0. W3C Recommendation. 1998, verfügbar unter http://www. w3.org/TR/1998/REC-xml-19980210

[Bux+01] Buxmann, P.; Martín, L.; Wüstner, E.: SIMPLEX - Eine prototypische SupplyChain-Management-Lösung für den Austausch, die Konvertierung und die Integration von XML-Dokumenten. In: Buhl, H. U.; Huther, A.; Reitwiesner, B. (Hrsg.) Information Age Economy. 5. Internationale Tagung Wirtschaftsinformatik. Physica, Heidelberg, 2001: S. 441-453.

[Fall01] Fallside, D.C.: XML Schema Part 0: Primer. W3C Recommendation. 2001, http://www.w3.org/TR/xmlschema-0, Abruf am 2004-06-06.

[HeSc04] Hess, T.; Schulze, B.: Mehrfachverwertung von Inhalten in der Medienindustrie. Grundlagen, Varianten und Herausforderungen. In: Altmeppen, K. D.; Karmasin, M.: Medien und Ökonomie. Band 2: Problemfelder der Medienökonomie. VS Verlag, Wiesbaden, 2004: S. 41-62.

[Mars01] Marshall, M.: XML - Like The Air That We Breathe, InformationWeek.com, March 5, 2001, http://www.informationweek.com/827/xml.htm, Abruf am 2004-06-06. 
[QuWi03] Quantz, L.; Wichmann, T.: E-Business-Standards in Deutschland. Bestandsaufnahme, Probleme, Perspektiven. Endbericht des Forschungsauftrags des Bundesministeriums für Wirtschaft und Arbeit. Berlecon Research GmbH, 2003.

[RaHe00] Rawolle, J.; Hess, T.: XML als Basis für einen zwischenbetrieblichen Inhalteaustausch in der Medienindustrie. In: Proceedings zur Tagung „1. Deutsche Tagung XML 2000“. Heidelberg, 2000: S. 25-33.

[RaHe01] Rawolle, J.; Hess, T.: XML in der Medienindustrie - Ökonomische Aspekte. In: Turowski, K.; Fellner, J. (Hrsg.) XML in der betrieblichen Praxis - Standards, Möglichkeiten, Praxisbeispiele. Dpunkt Verlag, Heidelberg, 2001: S. 229-244.

[Raw+02] Rawolle, J.; Ade, J.; Schumann, M.: XML als Integrationstechnologie bei Informationsanbietern im Internet - Fallstudie bei BertelsmannSpringer. In: Wirtschaftsinformatik 44 (2002) 1: S. 19-28.

[Rawo02] Rawolle, J.: Content Management integrierter Medienprodukte - Ein XMLbasierter Ansatz. Deutscher Universitäts-Verlag: Wiesbaden, 2002.

[Roge95] Rogers, E. M.: Diffusion of Innovations. 4. Auflage. The Free Press, New York, 1995.

[RoRi01] Rothfuß, G.; Ried, C.: Content Management mit XML. Berlin et al., 2001.

[ScHe02] Schumann, M; Hess, T.: Grundfragen der Medienwirtschaft. 2. Auflage. Springer-Verlag, Berlin et. al., 2002.

[Spe+02] Specht, G.; Beckmann, C.; Amelingmeyer, J.: F\&E-Management - Kompetenz im Innovationsprozess. 2. überarb. und erw.Auflage. Schäffer-Pöschel, Stuttgart, 2002.

[Wei+98] Weitzel, T.; Buxmann, P.; Ladner, F.; König, W.: XML - Konzept und Anwendungen der Extensible Markup Language. SFB 403 Forschungsbericht. 1998, verfügbar unter http://www.wi-frankfurt.de/projectb3/deu/publikat/xml/index.htm

[Wei+01a] Weitzel, T.; Buxmann, P.; Ladner, F.; Kronenberg, R.: XML und EDI - Der Stand der Standardisierung. In: Rossbach, G. (Hrsg.) Mobile Internet. Dpunkt Verlag, Karlsruhe, 2001, S. 269-283.

[Wei+01b] Weitzel, T.; Harder, T.; Buxmann, P.: Electronic Business und EDI mit XML. Dpunkt Verlag, Heidelberg, 2001.

[Wei+03] Weitzel, T.; Martin, S.V.; König, W.: Straight Through Processing auf XMLBasis im Wertpapiergeschäft. In: Wirtschaftsinformatik 45 (2003) 4: S. 409-420.

[Wirt03] Wirtz, B.W.: Medien- und Internetmanagement. Gabler, Wiesbaden, 2003. 